\title{
OLIGODONCIA: REPORTE DE UN CASO CLÍNICO
}

\author{
${ }^{1}$ Rodrigo Enrique Guzmán Lemus, ${ }^{2}$ Patricia Trejo Quiroz, ${ }^{2}$ Claudia Sofia de León Torres, \\ ${ }^{1}$ Daniela Carmona Ruiz. \\ ${ }^{1}$ Especialista en Odontopediatría DEPeI F. de Odontología U. Nacional Autónoma de México, México. \\ ${ }^{2}$ Académico Especialización en Odontopediatría F. de Odontología U. Nacional Autónoma de México, México.
}

\section{Autor responsable de correspondencia: Claudia Sofía de León Torres}

Correo electrónico: deleon@fo.odonto.unam.mex

\section{RESUMEN}

Las anomalías dentales son alteraciones que se producen durante la odontogénesis en la se afecta la forma, el tamaño, la disposición, el grado de desarrollo y el número de los dientes. Dentro de estas anomalías de número, la agenesia dental se presenta con frecuencia. La oligodoncia se caracteriza por la ausencia congénita de más de seis dientes y puede presentarse en la dentición primaria y permanente; se asocia comúnmente con síndromes o algunas alteraciones sistémicas. Los terceros molares son los dientes mayormente afectados, seguidos por los segundos premolares inferiores y los incisivos laterales superiores. Esta entidad es más común en mujeres y en el maxilar inferior. En el presente artículo se reporta el caso de una joven de 13 años de edad quien acude a la Clínica de Odontopediatría de la Universidad Nacional Autónoma de México con ausencia congénita de los órganos dentales del 12,17,18, 22, 27,28, 35, 37,38,45, 47 y 48. Dicha ausencia no se asoció con algún tipo de síndrome que tenga dentro de sus características la ausencia dental. Por tal motivo, se estableció un diagnóstico presuntivo de oligodoncia no sindrómica verdadera y se planteó llevar a cabo un tratamiento que cumplía con las necesidades que el caso requiera mediante la interacción multidisciplinaria de diferentes especialidades odontológicas. [Guzmán RE, Trejo P, De León C, Carmona D. Oligodoncia: reporte de un caso clínico. Ustasalud 2012; 11: 124 - 129]

Palabras clave: Anodoncia, Anomalía, Diente premolar

\section{OLIGODONTIA: A CASE REPORT}

\section{ABSTRACT}

Dental anomalies are disorders that can occur during odontogenesis (initiation and proliferation of the dental lamina),in which it affects the shape, the size, layout, degree of development and number of the teeth. Within these anomalies of number, dental agenesis one of those that occur most frequently within the population and there are several types among them: dental agenesis of one or more teeth that can be hypodontia (absence of less than six teeth), or oligodontia (absence of six or more teeth, excluding third molars). Oligodontia is a tooth developmental disorder characterized by congenital absence of more than six teeth and can occur in the primary and permanent dentition and it is commonly associated with certain systemic disorders or syndromes. The third molars are the most affected teeth within this disorder, followed by the second lower premolars and upper lateral incisors. It is usual in the female population and in the mandibular region. The present paper reports the case of a 13 year -old patient, who attended to the Pediatric Dental Clinic of the Universidad Nacional Autónoma de México with congenital absence of the following dental organs 12, 17, 18, 22, 27, 28, 35, 36, 37, 38, 45, 47 and 48. This absence was not associated with any type of syndrome. Therefore, a presumptive diagnosis of non-syndromic oligodontia was established and it was considered to perform a treatment by a multidisciplinary team of different dental specialities.

Key words: Anodontia, Hypodontia, Tooth agenesis

Recibido para publicación: enero 10 de 2012. Aceptado para publicación: septiembre 30 de 2012.

\section{INTRODUCCIÓN}

En los últimos años ha aumentado la frecuencia de oligodoncia al afectar a una gran parte de la población mundial. La oligodoncia se define como la ausencia congénita de más de seis dientes en la dentición primaria y/o permanente y es considerada como una anomalía del desarrollo dental que comúnmente, se encuentra asociada a síndromes y alteraciones sistémicas. ${ }^{1}$

Existen diversos tipos entre los cuales están la agenesia dental de uno o más dientes que puede ser hipodoncia si existe ausencia de menos de seis dientes, ${ }^{2}$ suele presentarse en forma aislada y, por lo general, no se asocia con otra anomalía. El otro tipo es la oligodoncia que se refiere a ausencia de seis o más dientes sin tener en cuenta los terceros molares, puede ser una entidad aislada en la que el paciente no presenta ninguna otra alteración asociada. En otras ocasiones, la oligodoncia puede estar asociada con algún síndrome y se vincula con defectos en piel, ojos, oídos y esqueleto lo que también se conoce como Agenesia Dental Selectiva (STHAG) que se considera una de las anomalías más comunes de la dentición humana. ${ }^{1-4}$

Algunos autores, clasifican la oligodoncia de la siguiente manera:

Verdadera o absoluta, que se da cuando no hay formación de ninguno de los gérmenes dentarios. ${ }^{5}$ 
Falsa o relativa, cuando clínicamente no se observan los dientes y con la radiografía se comprueba su presencia. ${ }^{3,6}$

Adquirida o inducida es consecuencia de la extracción de los dientes. ${ }^{3,4}$

Se ha reportado que la agenesia de dientes permanentes es del 10 al 25\% en la población americana con una prevalencia del 5 al 10\% en las poblaciones asiática y europea. Se presenta con mayor frecuencia en terceros molares (7\%), seguida de los segundos premolares inferiores (6\%) y en tercer lugar la de laterales superiores. $(4 \%))^{7,8}$ Se presenta en personas del sexo femenino en un $8,4 \%$ y en el masculino en un $6,5 \%,{ }^{9}$ observándose una mayor frecuencia de esta afección en la región mandibular.?

La oligodoncia como anomalía de número debe tenerse siempre presente por su etiología multifactorial, de ahí su posible diagnóstico clínico y radiográfico precoz. ${ }^{10}$ Desde temprana edad ( $41 / 205$ años de edad) es posible establecer la ausencia o presencia de todos los dientes, debido a que a esta edad la observación de los gérmenes dentales es viable en el examen radiográfico, con excepción de los terceros molares. Esta anomalía en el niño puede ocasionar afecciones tanto estéticas como funcionales, de ahí que sea necesario dirigir el tratamiento al tener en cuenta el patrón morfogenético del individuo y su posible rehabilitación mediante tratamientos ortodóncicos siempre que sean posibles por sus ventajas sobre los tratamientos protésicos. ${ }^{10-12}$

Esta alteración de número es el resultado de un trastorno en la iniciación y proliferación de la lámina dental, aproximadamente en la sexta semana de vida intrauterina, lo que impide la formación y diferenciación de las células que originan el germen dentario y provoca en este una atrofia. ${ }^{3,13}$ Varios son los factores que se atribuyen o asocian con este problema, por lo que se divide en dos grandes grupos, generales y hereditarios. Dentro de los factores causales generales de la oligodoncia se propone el traumatismo local, infección durante el desarrollo dental, quimioterapia o radioterapia, disfunción glandular, raquitismo, sífilis, sarampión durante el embarazo y disturbios intrauterinos severos, entre los más importantes. ${ }^{2,7}$ En lo referente a factores hereditarios se consideran las enfermedades sindrómicas que se relacionan con esta alteración.

La oligodoncia congénita parece ser el resultado de una o más mutaciones puntuales, a menudo transmitida con un patrón autosómico dominante. ${ }^{7}$ Estas mutaciones pueden ocurrir en los genes: MSX1, PAX9 (factor de transcripción juega un papel crítico en la odontogénesis), LTBP3.,11,14 Se ha demostra- do que la forma esporádica no sindrómica de oligodoncia dental familiar se asocia con mutaciones en MSX1 y productos PAX9. ${ }^{11}$

Se encuentra fuertemente asociada a síndromes que presentan expresiones variables. Tres son los síndromes característicos que comúnmente se relacionan con oligodoncia:

1. Displasia ectodérmica. Esta puede ser de tipo anhidrótica o hipohidrótica. La primera se presenta de una forma autosómica dominante y entre sus manifestaciones clínicas se encuentran: aplasia de glándulas sudoríparas, ausencia de glándulas sebáceas, pelo rubio, fino y escaso, falta de pestañas y cejas, pocos dientes en forma cónica. El tipo hipohidrótico se caracteriza por hipotricosis, hipoplasia malar, puente nasal ancho, labios invertidos, piel periorbital hiperpigmentada, orejas de inserción baja, piel delgada y múltiples dientes ausentes.

2. Síndrome óculo-mandíbulo-cefálico (Síndrome de Hallermann- Streiff). Es una alteración que se transmite tanto en forma autosómica recesiva como autosómica dominante, o se presenta a causa de una mutación "novo". Es una enfermedad poco usual puesto que solo se conocen 150 casos en todo el mundo. Presenta como características principales: microftalmia, enanismo, hipotricosis, hipoplasia maxilomandibular, escleróticas azules, microcefalia, dientes permanentes ausentes e hipodoncia de dientes primarios. Alrededor del $10 \%$ de los casos se acompañan de retraso neuro-motor.

3. Disgénesis mesoectodérmica. Los pacientes con esta alteración presentan cara amplia, deformidad de los ojos, distrofia muscular, premaxila subdesarrollada y algunas veces hipodoncia. $8,13,15,16$.

Otros síndromes que se encuentran relacionados son:

- Síndrome de Christ-Siemens

- Síndrome de Book

- Síndrome de Riegar

- Síndrome de dientes y uñas o Síndrome de Witkop

- Disostosis Cleidocraneal

- Síndrome de Down

- Disostosis Craneofacial

- Síndrome de Ellis van Creveld

- Síndrome de Gardner

- Disostosis Mandibulofacial. ${ }^{13,16,17 .}$

Un adecuado tratamiento protésico desempeña un papel de suma importancia en el manejo de los pa- 
cientes con oligodoncia en dentición temporal y/o permanente cuya dentadura no se desarrolla normalmente. La restauración de los espacios desdentados requiere la consideración de múltiples factores que incluyen el número de dientes perdidos, la distribución del espacio, tamaño de los dientes y la edad del paciente, entre los más importantes. ${ }^{10,16}$ Existen varias opciones para el tratamiento de la oligodoncia entre los que se encuentran: prótesis removibles metálicas, prótesis removibles de resina, prótesis fija e implantes dentales.

\section{REPORTE DE CASO}

Una paciente de sexo femenino con 13 años de edad, se presenta en la Clínica de Odontopediatría de la División de Estudios de Posgrado e Investigación (DEPeI) de la Facultad de Odontología de la Universidad Nacional Autónoma de México (UNAM). Acude por la malposición dental que presenta así como la persistencia de dientes temporales. En el interrogatorio de rutina no existen datos de relevancia para el diagnóstico del caso en los antecedentes heredofamiliares. En los antecedentes patológicos personales refiere que estuvo bajo tratamiento médico neurológico como consecuencia de síncopes frecuentes desde los 9 años, medicándola con Carbamazepina 100mg por día, descartado diagnóstico de epilepsia. Al momento del examen reporta que dichos síncopes no se presentan desde hace un año. Dentro de las enfermedades de la infancia, la paciente solo padeció varicela a los 6 años de edad.

En el examen clínico intraoral se observó presencia de dentición mixta, no correspondiente con la edad cronológica de la paciente. Se observó ausencia de los órganos dentales del 12, 15, 17, 22, 27, 35, 37, 45 y 47 y retención prolongada del 53, 55, 62, 63, 65 (Figura 1).

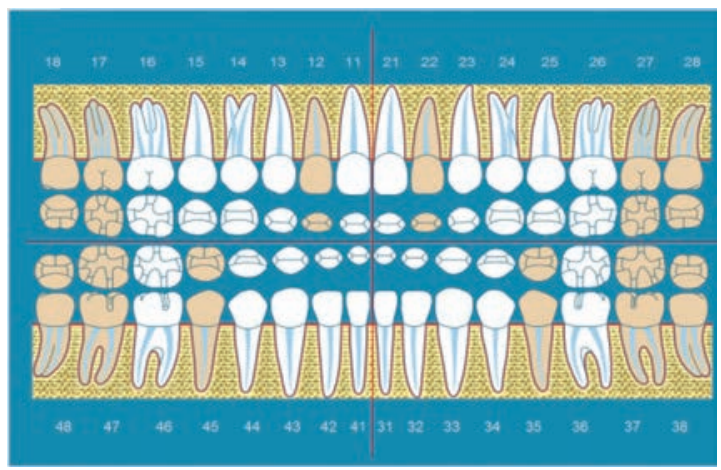

Figura 1. Odontograma de la paciente. En color amarillo se pueden observar los dientes ausentes congénitamente.

Se observó mordida cruzada posterior izquierda, malposición dental y se presumía la posible presencia de hábito de respiración bucal. Presentaba una higiene buena, realizaba el cepillado dental dos veces al día (Figuras 2, 3, 4, 5 y 6).

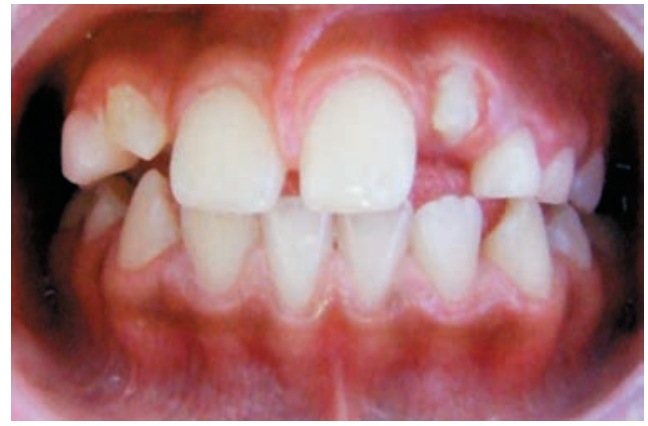

Figura 2. Vista frontal intraoral

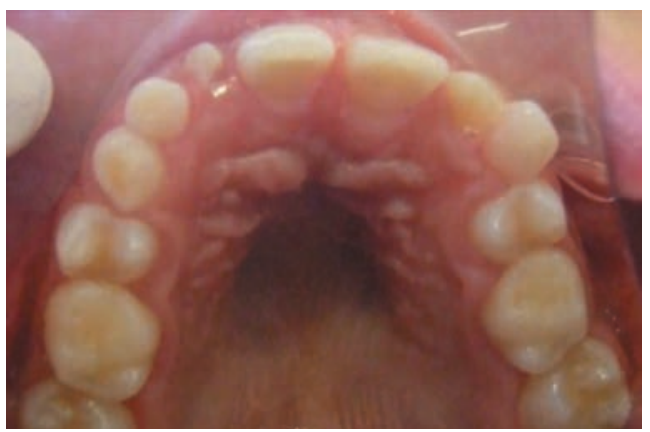

Figura 3. Vista del arco superior

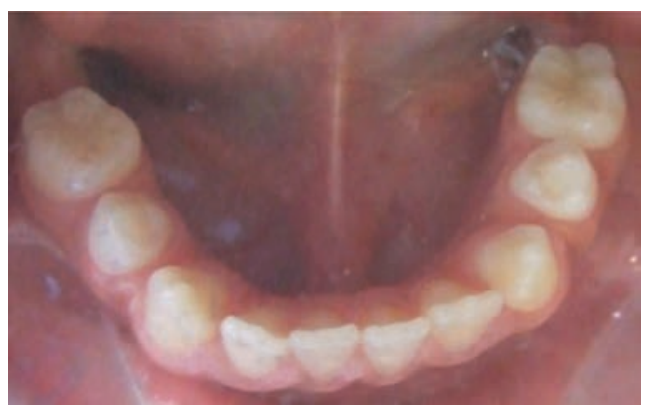

Figura 4. Vista del arco inferior

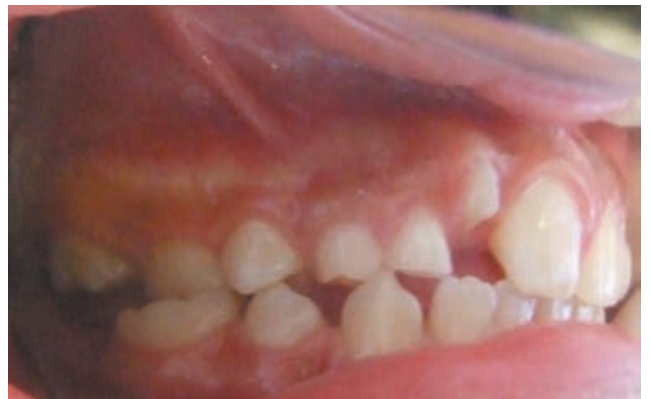

Figura 5. Vista lateral izquierda, se observa mordida cruzada posterior

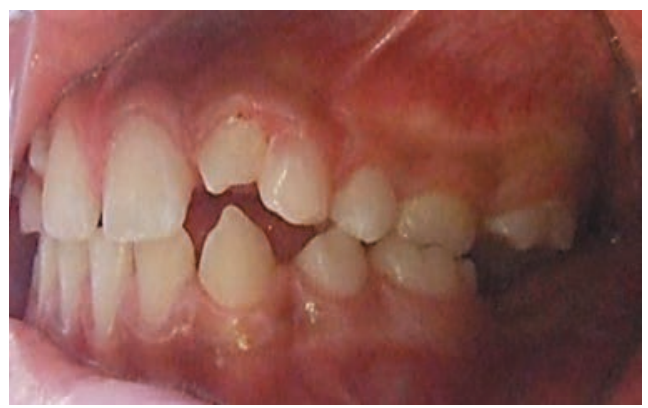

Figura 6. Vista lateral derecha 
Radiográficamente, se confirmó la ausencia de los órganos dentarios del 12, 17, 18, 22, 27, 28, 35, 37, $38,45,47$ y 48 . Es importante recordar que los terceros molares no son considerados dentro del conteo para poder definir la alteración como "Oligodoncia" como lo menciona en su trabajo Díaz y colaboradores; ${ }^{10}$ sin embargo, se puede observar que tampoco se encuentran presentes radiográficamente (Figura 7).

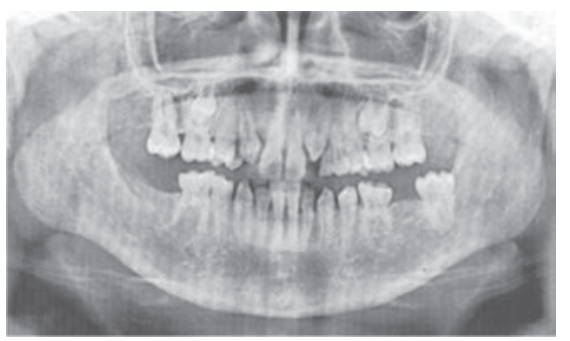

Figura 7. Radiografia panorámica, se observa la ausencia de múltiples órganos dentales.

Se descartó que la oligodoncia que presentaba la paciente estuviera relacionada con alguno de los síndromes asociados a ésta, debido a que no existían signos o síntomas de alteraciones de los tejidos derivados del ectodermo: piel, pelo, uñas y glándulas sudoríparas, entre otros. En la inspección clínica se pudo observar que la piel no presentaba alteraciones como sequedad o descamación; el cabello, las cejas, las pestañas, la piel de las manos y las uñas eran normales (Figuras 8, 9, 10, 11 y 12).

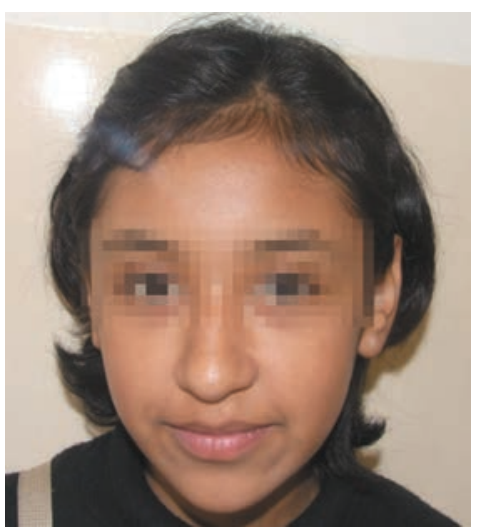

Figura 8. Fotografia frontal

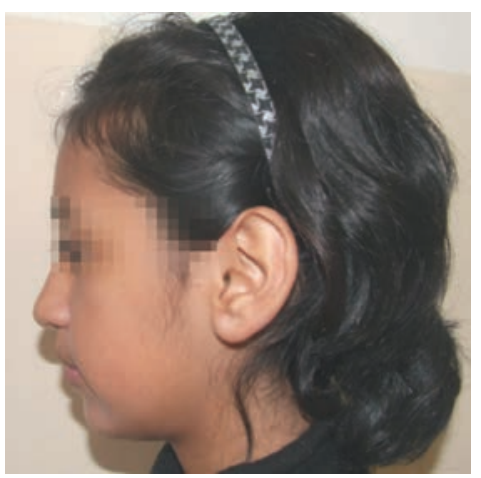

Figura 9. Perfil izquierdo

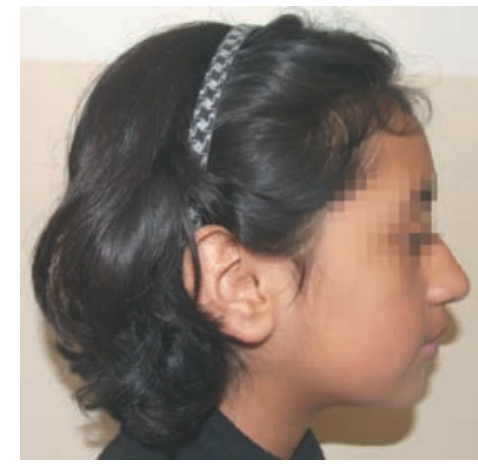

Figura 10. Perfil derecho

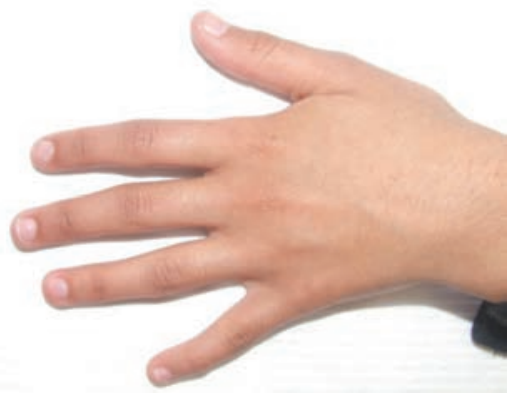

Figura 11. La piel y uñas de las manos son normales

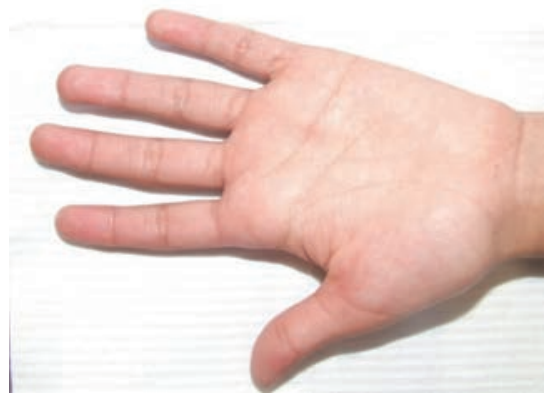

Figura 12. Fotografía de la palma de la mano izquierda, no se observan alteraciones relacionadas con algún síndrome

Con estas características, aunque no se contaba con una confirmación genética, se propuso un diagnóstico presuntivo de oligodoncia no sindrómica de tipo verdadera o absoluta.

El tratamiento planteado a la paciente constaba de tres fases:

- Fase 1. Protésica inicial o temprana, cuyo objetivo fue devolver la función y conservar el espacio al elaborar una prótesis removible provisional que sustituyera los múltiples órganos dentales ausentes.

- Fase 2. Ortopédico-Ortodóncico, con el fin de proporcionar una buena alineación y nivelación dental.

- Fase 3. Protésica final, en la que se presentarán como plan de tratamiento la opción de la fabricación de una prótesis removible definitiva o la colocación de implantes dentales, considerado para estos últimos, que es necesario llevar a cabo una 
evaluación de la condición de salud general de la paciente en la que se tomaría en cuenta enfermedades sistémicas, factores psicológicos, hábitos del paciente, bruxismo, higiene bucal y presencia de enfermedad periodontal, entre otros. De igual manera, se tomarían las consideraciones anatómicas pertinentes como son la localización del seno maxilar, localización del canal del nervio alveolar, condición de la encía adherida, grosor del hueso alveolar, defectos óseos o presencia de patologías en el sitio del implante.

En cuanto a la edad recomendada para llevar a cabo este tipo de tratamiento, aunque la literatura hace referencia que este rubro no es factor determinante a la hora de ser candidato para la colocación de implantes dentales, es recomendable no hacerlo antes de los 17 años, hasta que el crecimiento maxilomandibular se haya completado.

Lo anteriormente mencionado se llevaría a cabo con el objetivo de lograr una mayor funcionalidad, comodidad y estética para la pacientes así como mejorar su calidad de vida.

\section{DISCUSIÓN}

Uno de los aspectos que jugó un papel importante en este reporte de caso fue la interacción interdisciplinaria que se llevó a cabo para plantear el tratamiento como lo describe Sánchez y Moya en su trabajo, en el que mencionan que la oligodoncia no asociada a síndromes, no sólo ocasiona problemas funcionales y estéticos, sino también problemas psicológicos, especialmente para los jóvenes. ${ }^{18}$ Para esta paciente era evidente la existencia de una afectación psicológica marcada, puesto que su forma de comportarse y de relacionarse con las demás personas era de una manera tímida e insegura, al tratar en lo posible de ocultar su condición bucodental, por lo cual se consideró la necesidad de una terapia psicológica. El tratamiento de estos pacientes necesita de un enfoque multidisciplinario, que incluye pero no se limita a una colaboración entre el odontólogo, odontopediatra, ortodoncista y rehabilitador oral, así como en ocasiones, el psicólogo y terapista de lenguaje dada las peculiaridades anatómicas y los trastornos del desarrollo psíquico y físico que presentan. ${ }^{17}$

Gunbay y colaboradores proponen de igual manera, una estrategia de tratamiento que consiste en un enfoque interdisciplinario en equipo con fases ortodóncicas, quirúrgicas y prostodóncicas. Después de un tratamiento con ortodoncia fija, se considera un aumento de la cresta alveolar y posterior colocación de implantes dentales. En este caso, el tratamiento fue dividido en tres fases que proporcionaran de una manera más práctica satisfacer las necesidades de funcionalidad principalmente, y en segundo lugar una mayor estética. Estos puntualizan la importancia de una intervención odontológica precoz para mejorar el aspecto del paciente y minimizar el impacto emocional y psicosocial, ${ }^{19}$ sin embargo, no siempre se da la importancia que la situación amerita y se busca atención a edades avanzadas, donde ya es necesaria la intervención de tratamientos que ayuden en el aspecto psicosocial.

Por su parte, Ponce y colaboradores hacen referencia a la importancia del trabajo interdisciplinario entre el odontólogo, ortodoncista, odontopediatra, pediatra, genetista, cirujano máxilofacial, ortopedista, rehabilitador y todos aquellos especialistas que puedan colaborar para modificar y armonizar las alteraciones sindrómicas para restaurar la función y la estética, lo que permitirá que la persona afectada pueda hacer una vida lo más normal posible. ${ }^{15} \mathrm{El}$ tratamiento que se planteó en este caso, involucró las especialidades de odontopediatría, ortodoncia y rehabilitación oral, las cuales en conjunto, hicieron propuestas para poder llevar a cabo un tratamiento más completo; de igual manera, como se mencionó anteriormente, apoyándose en las diferentes disciplinas médico-estomatológicas, se busca brindar un tratamiento integral en este tipo de anomalía.

\section{Conclusiones}

La oligodoncia es una alteración de número que se caracteriza por la ausencia congénita de más de seis dientes y puede presentarse en la dentición primaria y/o permanente. Comúnmente, se presenta como característica de síndromes y en ocasiones como parte de alteraciones sistémicas, sin embargo, en raras ocasiones no asocia a factores genéticos, como es el caso que se refiere. Afecta principalmente a los terceros molares, segundos premolares, laterales superiores pero puede afectar cualquier otro órgano dental.

Es de vital importancia hacer un diagnóstico temprano y certero de esta entidad. Para ello, el apoyo en diferentes auxiliares de diagnóstico, como son radiografias panorámicas, periapicales y oclusales, estudios genéticos, y si el caso lo ameritara tomografías o estudios más específicos con el fin de proponer un tratamiento multidisciplinario adecuado así como dar, explicar y orientar adecuadamente a los pacientes y sus familiares.

\section{BIBLIOGRAFÍA}

1. Ulusu T, Cinar C, Nalbant D. Oligodontia associated whit femoral bifurcation, tibia hemimelia and cleft hand. Chin Med J (Engl) 2004; 117 (5): 787-788.

2. Noor A, Windpassinger C, Vitcu I, Orlic M, Rafiq MA, et al. Oligodontia is caused by mutation in LTBP3, the gene encoding latent TGF-b binding protein 3. Am J Hum Genet 2009; 84 (4). $519-523$.

3. Ponce-Bravo S, Ledesma-Montes C, Pérez-Pérez G, Sánchez-Acuña G, Morales-Sánchez I et al. Anodoncia no sindrómica. Estudio clínico-radiográfico. Revista ADM 2004; LXI (5): 171- 181. 
4. Stanley J. Diccionario Ilustrado de Odontología. Buenos Aires: Editorial Médica Panamericana; 1992.

5. Sheider E. Anodoncia completa de la dentición permanente. Reporte de un caso. Comp Ed Cont 2000; 6 (4): 7 - 10.

6. Reggezi JA. Patología Bucal. México. Editorial Interamericana; 1991.

7. Carbajal EE, Martínez BAM, Osende NH, Vázquez DJ. Oligodoncia: estudio radiográfico de un caso clínico. Acta Odontol Venez 2008 [en línea]; 46 (3). URL disponible en: http://www.scielo.org.ve/scielo.php?script = sci arttext\&pid $=$ S0001-63652008000300019

8. Reggezi JA, SciubbaJJ, Jordan RCK. Oral Pathology: Clinical Pathologic Correlations. 4th edition. St Louis: Elsevier Saunders; 2002.

9. Moret Y, González JM. Anodoncia Parcial relacionada con quiste dentígero y quiste traumático. Revisión de la literatura y presentación de un caso. Revista Odontológica. Venezolana 2003 [en línea]; 41 (3). URL disponible en: http:// www.scielo.org.ve/scielo.php?pid $=$ s00016365200 3000300007\&script $=$ sci_arttext.

10. Díaz JE, Fariñas M, Suárez AL. Prevalencia de oligodoncia en pacientes con tratamiento de ortodoncia. Facultad de Ciencias Médicas "Mariana Grajales Coello". Correo Científico Médico de Holguín 2000; 4 (4) [en línea]. URL disponible en: http://www.cocmed.sld.cu/no44/n44ori3.htm

11. Mostowska A, Biedziak B, Trzeciak WH. A novel mutation in PAX9 causes familial form of molar oligodontia. Eur J Hum Genet 2006; 14 (2): 173-179.

12. Sakai VT, Oliveira TM, Pessan JP, Santos CF, Machado MA. Alternative oral rehabilitation of children with hypodontia andconical tooth shape: a clinical report". Quintessence Int 2006;37 (9):725 - 730.

13. Schalk-van derWeide Y, Steen WH, Bosman F. Distribution of missing teeth and tooth morphology in patients with oligodontia. ASDC J Dent Child 1992; 59 (2): 133- 140.

14. Kim JW, Simmer JP, Lin BP, Hu JC. Novel MSX1 frameshift causes autosomal-dominant oligodontia. J Dent Res 2006; 85 (3): $267-271$.

15. Ponce S, Morales I, Ledesma C, Garcés M. Síndromes vinculados con la anodoncia. Revisión de la literatura. Revista ADM 2010; 67 (2): 62- 64.

16. Chung DW, Vang MS, Park SW, Lim HP, Yang HS.Oral rehabilitation for a patient with oligodontia and maxillary hypoplasia. J Adv Prosthodont 2009; 1 (1): 6 - 9.

17. Symons AL, Stritzel F, Stamation J. Anomalies associated whithhypodontia of the permanent lateral incisor and second premolar. J Clin Pediatric Dent 1993; 17 (2): 109 - 111.

18. Sánchez A, Moya MJ. Revisión de la oligodoncia no sindrómica y presentación de 18 casos. Medicina Oral Patología Oral y Cirugía Bucal 2009; 14 (4): 217 - 220.

19. Gubay T, Özveri B, Sipahi A, Bulut H, Dundar M. Enfoque multidisciplinario en un paciente con oligodoncia no sindrómica utilizando técnicas quirúrgicas avanzadas. Revista Internacional de Odontología Restauradora \& Periodoncia 2011; 15 (3): 296 - 305.

Correos electrónicos de los autores:

Rodrigo Enrique Guzmán Lemus: rodrigo_egl@hotmail.com Patricia Trejo Quiroz: pichonx3m@gmail.com Claudia Sofia de León Torres: deleon@fo.odonto.unam.mex Daniela Carmona Ruiz: danielacarmona@hotmail.com
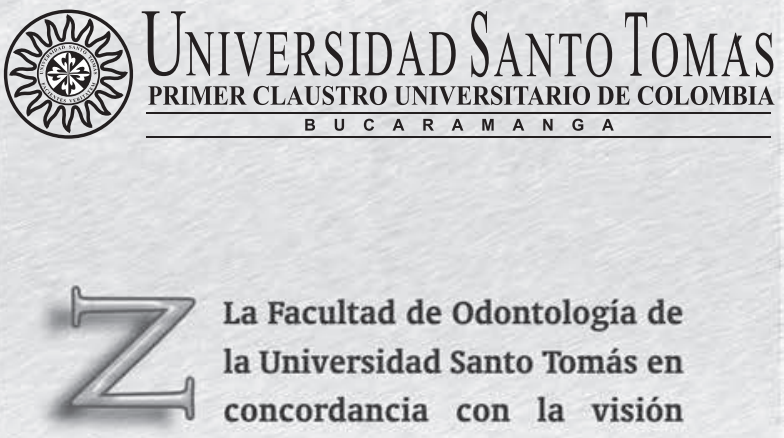

La Facultad de Odontología de la Universidad Santo Tomás en concordancia con la visión institucional aspira lograr:

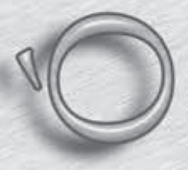

Un compromiso efectivo de la comprensión renovada del hombre colombiano, su mundo y sus valores, en un contexto de salud integral para todos; el reconocimiento nacional e internacional, como comuni-

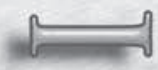
dad académica, a través de la investigación, la producción intelectual y el intercambio académico; liderazgo en los procesos relacionados con la salud bucal de la comunidad, a nivel regional y nacional; la cualificación permanente de sus servicios a la comunidad para responder efectiva $\mathbf{y}$ oportunamente a sus usuarios; el fortalecimiento permanente de su accionar pedagógico mediante el desarrollo humano integral, la aplicación de tecnologias de punta y la proyección comunitaria. 